\title{
New complex bearing end-to-end dicyanamide as a double bridging ligand: Crystallography study and magnetic properties
}

\author{
Abderazak Addala $^{a^{*}}$, Fatima Setifi ${ }^{\mathrm{a}}$, Zouaoui Setifi $^{\mathrm{a}}$, Piotr Konieczny $^{\mathrm{b}}$ and Robert Pelka ${ }^{\mathrm{b}}$ \\ ${ }^{a}$ Laboratoire de Chimie, Ingénierie Moléculaire et Nanostructures (LCIMN), Université Ferhat Abbas- Sétif 1, 19000 \\ Sétif, Algeria. \\ ${ }^{\mathrm{b}}$ Institute of Nuclear Physics, Polish Academy of Sciences, Radzikowskiego 152, 31-342 Krakow, Poland
}

\begin{abstract}
A new polymeric, two-dimensional compound $\left[\mathrm{Fe}\left(4,4^{\prime}\right.\right.$-bpy $\left.)\left(\mu_{1,5} \text {-dca }\right)_{2}\right] \cdot 0 \cdot 24\left(4,4^{\prime}-\right.$ bpy $) \cdot 0 \cdot 5(\mathrm{EtOH})(\mathbf{1})$, in which dca= dicyanamide and 4,4'-bpy $=4,4^{\prime}$-bipyridine, has been synthesized under solvothermal conditions and fully characterized by elemental analysis, FT-IR spectroscopy and X-ray single crystal diffraction. Structural analysis of compound $\mathbf{1}$ showed it is a novel two dimensional coordination polymer, constructed from repetition of 4,4'-bpy and dca as a bidentate bridging ligand. Using the co-ligand 4,4'-bpy, lead to completely different structure geometry and network, which confirms the substantial effect of co-ligand. Furthermore, magnetic properties have been performed to understand the correlation structure-properties.
\end{abstract}

Keywords: Structure, Coordination polymer, Iron complex, Dicyanamide; Double bridge; magnetic properties.

\section{Introduction}

The field of crystal engineering of metal-organic structures in order to obtain compounds with various multidimensional networks is of particular modern researches [1]-[4].

Transition metal compounds with $\mathrm{N}$-donor bridging ligands are used to form infinite polymeric frameworks[3]. These compounds display rich structural and topological features and exhibit physical properties ranging from conductivity to magnetism[4].

Recently, polynitrile and pseudohalide ligands, have proven to be very versatile and diverse in both coordination chemistry and in molecular materials chemistry [5-16]. In particular, the pseudohalide ligand dicyanamide, hereafter dca, with the formula of $\left[\mathrm{N}(\mathrm{CN})_{2}\right]$ received considerable attention because of its various modes of coordination character and bridging ability. It is a well-known building block to create 1D and 2D frameworks owing to its flexibility and versatility in coordination modes. Also dca is an anionic ligand, so no other anions are required in the structure to balance the charge[17].

The various coordination and bridging modes of dca as a ligand are illustrated by its four distinct structures in Scheme 1. Part (I) shows monodentate coordination through a nitrile nitrogen atom, (II) bidentate $\left(\mu_{2}\right)$ end-to-end bridging through the two nitrile nitrogen atoms, (III) tris-monodentate $\left(\mu_{3}\right)$ bridging of three metal atom and (IV), which is very similar to that of (II), except that a pair of dca ligands makes a double bridge between two metal centers.

The third coordination mode, $\mu_{3}$, observed in several neutral 3D compounds with the general formula $\left[\mathrm{M}^{\mathrm{II}}(\mathrm{dca})_{2}\right]$ in which $\mathrm{M}(\mathrm{II})$ is $\mathrm{Fe}, \mathrm{Mn}, \mathrm{Co}, \mathrm{Ni}$ or $\mathrm{Cu}$, which exhibit a rutile-like structure [3], [4], [18], [19].

Lower dimensional systems with general formula of $\left[\mathrm{M}(\mathrm{L})_{2}(\mathrm{dca})_{4}\right]$ where $\mathrm{L}$ is monodentate ligand, have also been reported by coordination of two blocking L ligands. In this case the four remaining coordination sites are occupied by the nitrogen atoms of the dca ligands, leading to square twodimensional systems with each metal center bridged by means of four single end-to-end dca to four 
neighboring ones [18], [20], [21], [22] or one-dimensional systems with double end-to-end dca bridges along the chains [23], [24].

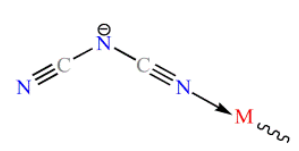

(I)

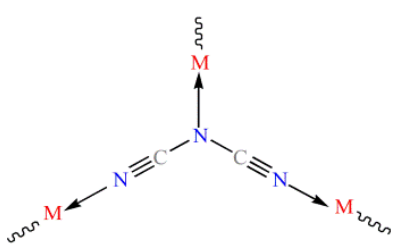

(III)

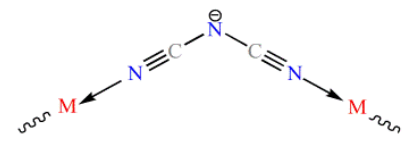

(II)

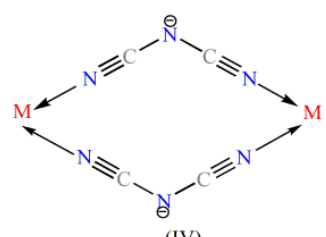

Scheme 1.Different coordination modes of dca as single bridging ligand in:(I) mondentate, (II) bidentate, (III) tridentate and as double bridging mode in (IV).

The structures and networks of the coordination polymers of dca can be strongly modified by the introduction of co-ligands[25]. In this research we focused on the N-donor ligand (4,4'-bpy), as coligand. A novel polymeric network structure based on iron complexe of dca was synthesized successfully. Using dca along with 4,4'-bpy ligand leads to polymeric planes in compound 1.

\section{Experimental}

\subsection{Reagents and techniques}

All the reagents and solvents employed were commercially available and used as received without further purification. Elemental analyses $(\mathrm{C}, \mathrm{H}$ and $\mathrm{N})$ were performed using a Perkin-Elmer 2400 series II CHN analyzer. Infrared spectra were recorded in the range of $4000-500 \mathrm{~cm}^{-1}$ on a FT-IR Bruker ATR Vertex 70 Spectrometer.

\subsection{X-ray crystallography}

Diffraction data were collected at $200 \mathrm{~K}$ for 1, using a Bruker APEXII CCD diffractometer and graphite-monochromated MoK $\alpha$ radiation $(\lambda=0.71073 \AA)$, using a Bruker SMART X2S diffractometer and monochromated (doubly curved silicon crystal) MoK $\alpha$ radiation $(\lambda=0.71073$ $\AA$ ). Data collection, cell refinement and data reduction were performed using APEX2 and SAINT [30], and absorption correction with SADABS [30]. The structures were solved by direct methods using SHELXS-97 [31] and refined by full-matrix least squares on $F^{2}$ with all data, using SHELXL2014 [32]. All $\mathrm{H}$ atoms were located in difference maps: those bonded to $\mathrm{C}$ atoms were treated as riding atoms in geometrically idealized positions with $\mathrm{C}-\mathrm{H}$ distances $0.95 \AA$ (pyridyl), $0.98 \AA$ $\left(\mathrm{CH}_{3}\right)$ or $0.99 \AA\left(\mathrm{CH}_{2}\right)$ and with $U_{\text {iso }}(\mathrm{H})=\mathrm{k} U_{\text {eq }}(\mathrm{C})$. where $k=1.2$ for all $\mathrm{H}$ atoms bonded to $\mathrm{C}$ atoms. The ethanol components was found to be disordered over two sets of atomic sites having unequal occupancies: for the minor component, the bonded distances and the 1,3 non-bonded C...O distances were restrained to be the same as those in the major component, subject to S.U.s of 0.005 $\AA$ and $0.01 \AA$ respectively.In addition, the anisotropic displacement parameters for pairs of partialoccupancy atoms occupying essentially the same physical space were constrained to be identical. For the $\mathrm{H}$ atoms bonded to $\mathrm{O}$ atoms, the atomic coordinates were refined with $U_{\text {iso }}(\mathrm{H})=1.5 U_{\text {eq }}(\mathrm{O})$ giving $\mathrm{O}-\mathrm{H}$ distances of $0.840(10) \AA$, and the occupancies of the ethanol components refine to values of 0.694(9) and 0.306(9), while the occupancy of the uncoordinated 4,4'-bipy unit refined to 


\section{A. ADDALE et al./ISITES2018 Alanya - Antalya - Turkey}

0.963(6).Crystallographic data and details of the data collection and structure solution and refinements are listed in Table 1.

Table 1.Crystal data and structure refinement parameters of complexe 1.

\begin{tabular}{|c|c|}
\hline & 1 \\
\hline Chemical formula & $2\left(\mathrm{C}_{14} \mathrm{H}_{8} \mathrm{FeN}_{8}\right) \cdot 0 \cdot 4815\left(\mathrm{C}_{10} \mathrm{H}_{8} \mathrm{~N}_{2}\right) \cdot \mathrm{C}_{2} \mathrm{H}_{6} \mathrm{O}$ \\
\hline$M_{\mathrm{r}}$ & 809.54 \\
\hline Crystal system, space group & Monoclinic, $C 2 / m$ \\
\hline Temperature (K) & 200 \\
\hline$a, b, c(\AA)$ & $19.908(9), 14.944(8), 12.194(6)$ \\
\hline$\alpha, \beta, \gamma\left(^{\circ}\right)$ & 90, $91.379(18), 90$ \\
\hline$V\left(\AA^{3}\right)$ & $3627(3)$ \\
\hline$Z$ & 4 \\
\hline Radiation type & Mo K $\alpha$ \\
\hline$\mu\left(\mathrm{mm}^{-1}\right)$ & 0.856 \\
\hline Crystal size (mm) & $0.50 \times 0.50 \times 0.30$ \\
\hline Diffractometer & Bruker SMART X2S \\
\hline Absorption correction & $\begin{array}{c}\text { Multi-scan } \\
(S A D A B S ; \text { Bruker, 2013) }\end{array}$ \\
\hline$T_{\min }, T_{\max }$ & $0.815,0.843$ \\
\hline $\begin{array}{l}\text { No. of measured, independent } \\
\text { and observed }[I>2 \sigma(I)] \\
\text { reflections }\end{array}$ & $15510,3794,2246$ \\
\hline$R_{\text {int }}$ & 0.047 \\
\hline$(\sin \theta / \lambda)_{\max }\left(\AA^{-1}\right)$ & 0.625 \\
\hline$R\left[F^{2}>2 \sigma\left(F^{2}\right)\right], w R\left(F^{2}\right), S$ & $0.043,0.125,1.04$ \\
\hline .....No. of reflections & 3794 \\
\hline No. of parameters & 280 \\
\hline No. of restraints & 65 \\
\hline $\mathrm{H}$-atom treatment & H-atom parameters constrained \\
\hline$\Delta \rho_{\max }, \Delta \rho_{\min }\left(\mathrm{e} \AA^{-3}\right)$ & $0.66,-0.41$ \\
\hline
\end{tabular}

\subsection{Synthesis of $\left[\mathrm{Fe}\left(4,4^{\prime}\right.\right.$-bpy $\left.)\left(\mu_{1,5} \text {-dca }\right)_{2}\right] \cdot 0.24(4,4$ '-bpy $) \cdot 0.5(\mathrm{EtOH})(\mathbf{1})$}

A mixture of $\mathrm{FeSO}_{4} .7 \mathrm{H}_{2} \mathrm{O}(0.2 \mathrm{mmol}, 56 \mathrm{mg}), 4,4$ '-bpy $(0.2 \mathrm{mmol}, 31 \mathrm{mg})$ and Nadca $(0.4 \mathrm{mmol}$, $36 \mathrm{mg})$ in $\mathrm{H}_{2} \mathrm{O} / \mathrm{EtOH}(1: 1 \mathrm{v} / \mathrm{v}, 10 \mathrm{~mL})$ was heated at $453 \mathrm{~K}$ for 2 days in a sealed Teflon-lined stainless steel vessel under autogenous pressure and then gradually cooled to room temperature at a rate of $10 \mathrm{~K} \mathrm{~h}^{-1}$. After the reaction vessel was cooled to ambient temperature. Orange crystals of 1 were obtained with a yield of 20\%. Anal.Calcd.for 1: C, 51.66; H, 3.22; N, 29.34. Found: C, 51.92; H, 3.19; N, 30.43\%. Selected IR bands $\left(v / \mathrm{cm}^{-1}\right): v_{\mathrm{s}}(\mathrm{C} \equiv \mathrm{N}) 2187(\mathrm{vs}) ; v_{\mathrm{as}}(\mathrm{C} \equiv \mathrm{N}) 2244(\mathrm{~m}) ; v_{\mathrm{as}}(\mathrm{C} \equiv \mathrm{N})+$ $v_{\mathrm{s}}(\mathrm{C} \equiv \mathrm{N}) 2307(\mathrm{~m})$.

\section{Results and discussion}

\subsection{Infrared spectroscopy}

The IR spectra of the dicyanamido compound exhibit strong to medium absorptions in the 2320$2110 \mathrm{~cm}^{-1}$ region corresponding to the $v(\mathrm{C} \equiv \mathrm{N})$ stretching frequencies of the dca ligand. In the complexe, the frequencies are shifted to higher values compared to the free dca. These results are in agreement with the literature for coordinated dca ligand [33]. 


\subsection{Description of the crystal structures $\left[\mathrm{Fe}\left(4,4^{\prime}-b p y\right)\left(\mu_{1,5}-d c a\right)_{2}\right]_{\mathrm{n}} \cdot 0.24\left(4,4^{\prime}-b p y\right) \cdot 0.5(E t O H)(1)$}

Complex 1 (perspective view is shown in Fig. 1 and selected bond lengths and bond angles are summarized in Table 2) crystallizes as a two-dimensional coordination polymer in space group $\mathrm{C} 2 / \mathrm{m}$. One pair of trans coordination sites at the octahedral Fe centre is occupied by two independent 4,4'-bipy ligands. One of these lies across the two-fold rotation axis along $(1 / 2, y, 0)$ and so bridges the two Fe centres at $(x, y, z)$ and $(1-x, y, 2-z)$, while the other lies across the twofold rotation axis along $(0, y, 1 / 2)$, so linking the Fe centres at $(x, y, z)$ and $(-x, y, 1-z)$. In this way a chain of composition $[\mathrm{Fe}(4,4 \text { '-bipy })]_{\mathrm{n}}$ is formed, running parallel to the [101] direction. The other four coordination sites are occupied by the terminal $\mathrm{N}$ atoms of four independent dca ligands, all of which lie across mirror planes. One pair of dca units, occupying mutually cis sites lie across the mirror plane at $y=0$, so linking the Fe centres at $(x, y, z)$ and $(x,-y, z)$, while the second pair lie across the mirror plane at $y=0.5$, linking the Fe centres at $(x, y, z)$ and $(x, 1-y, z)$. These interactions form a chain of spiro-fused 12-membered rings of composition $\left[\mathrm{Fe}(\mathrm{dca})_{2}\right]_{\mathrm{n}}$ running parallel to the [010] direction. The combination of the [010] and [101] chains generates an almost planar sheet, in the form of a $(4,4)$ net lying parallel to $(10-1)$ and having composition $\left[\mathrm{Fe}\left(4,44^{\prime}-\text { bipy }\right)(\mathrm{dca})_{2}\right]_{\mathrm{n}}(\mathrm{Fig}$. 2).

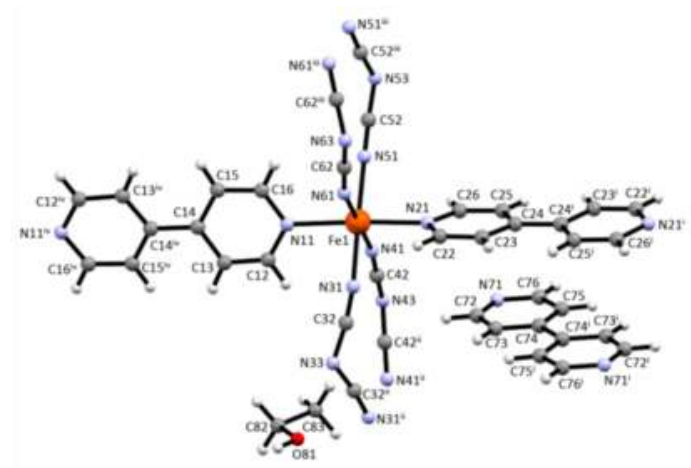

Fig. 1. Perspective view of a section of 1 together with partial atom numbering. Symmetry codes: (i) $-x, y,-z+1$; (ii) $x,-y, z$; (iii) $x,-y+1, z$; (iv) $-x+1, y,-z+2$.

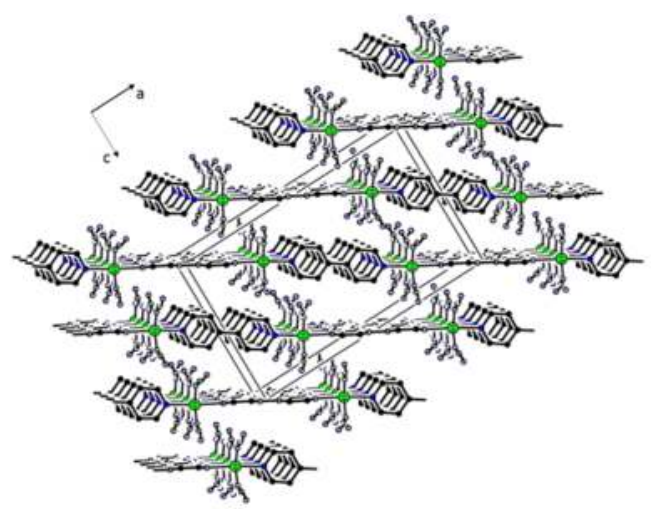

Fig. 2. 2-D network structure of complex 1 in the $a c$-plane.H atoms, ethanol molecules and free bpy ligands have been omitted for clarity.

Two sheets of this type, related to one another by the C-centring operation, pass through each unit cell, but these sheets occupy only ca. $75 \%$ of the available volume of the unit-cell. The sheets enclose two pairs of voids, one pair centred at $(0,0,1 / 2)$ and $(1 / 2,1 / 2,1 / 2)$ each having a volume of ca. 
$250 \AA^{3}$, and a second pair centred at $(1 / 2,0,1 / 2)$ and $(0,1 / 2,1 / 2)$ with a volume of ca. $215 \AA^{3}$. Each of the larger voids is occupied by an uncoordinated 4,4'-bipy unit, lying on a site of $2 / \mathrm{m}\left(C_{2 \mathrm{~h}}\right)$ symmetry and having occupancy $0.963(6)$. Each of the smaller voids is occupied by an inversionrelated pair of ethanol molecules, each disordered over two sets of atomic site having occupancies $0.694(9)$ and $0.306(9)$. Hence the overall composition of this material is $4\left[\mathrm{Fe}\left(4,4^{\prime}-\right.\right.$ bipy)(dca $\left.)_{2}\right] \cdot 0 \cdot 963(4,4$ '-bipy).2(EtOH).

The composition of the coordination polymer sheets indicates that iron is present as $\mathrm{Fe}(\mathrm{II})$, and the Fe-N distances indicate that the Fe(II) adopts a HS configuration. Thus, the Fe-N(4,4'-bipy) distances 2.209(2) $\AA$ and 2.227(2) $\AA$ are close to Fe-N(pyridyl) distances in other HS complexes [34], [35], and the Fe-N(dca) distances, which are closely clustered around $2.15 \AA$ are of similar magnitude to the Fe-N(4,4'-bipy) distances here. By contrast, in low-spin Fe(II) complexes of 2,2'bipy and 1,10-phen, the Fe-N distances are all close to $1.97 \AA$ [36]-[38]. For comparison, an analysis of interatomic distances in simple salts indicated a difference of ca, $0.16 \AA$ in effective radius between HS octahedral Fe(II) and the corresponding LS counterpart [39].

Within the sheets, the two independent Fe...Fe distances bridged by the dca ligands are 7.447(3) $\AA$ and 7.497(3) $\AA$, while those across the 4,4'-bipy bridges are 11.533(4) $\AA$ and 11.583(4) $\AA$ (Fig. 3).

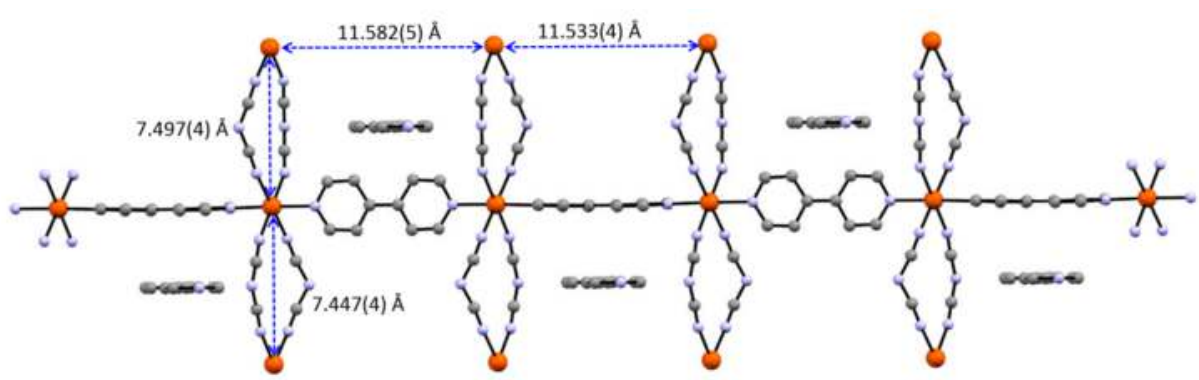

Fig.3. 1-D chain extension along the [101]. Ethanol molecules and hydrogen atoms are omitted for clarity.

\subsection{Magnetic Properties}

\subsubsection{Experimental}

To get insight into the magnetic properties of the reported sample (1) the measurements of the isothermal magnetization and susceptibility of a bulk sample have been performed. The mass of $3.7 \mathrm{mg}$ of the powder sample of $\mathbf{1}$ was mounted in a plastic draw in the Quantum Design SQUID probe. The magnetization was detected at $2.0 \mathrm{~K}$ in the field range of $-70-70 \mathrm{kOe}$ (black symbols in Fig. 5). The susceptibility was measured using the dc field of 500 Oe in the temperature range 2$300 \mathrm{~K}$ (black symbols in Fig. 4).

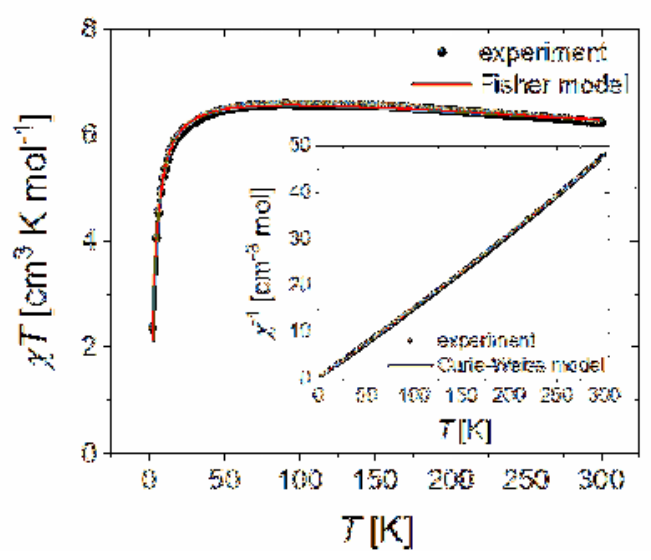

Fig. 4. Temperature dependence of the $\chi T$ of the powder sample of 1 . The red solid lineshows the best fit of the Fisher model. Inset: Temperature dependence of the inverse susceptibility of $\mathbf{1}$. The red solid line shows the best fit to the Curie-Weiss model appended with an additive correction. 


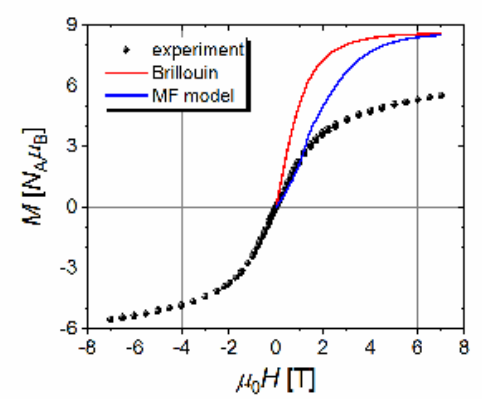

Fig. 5. Field dependence of the isothermal magnetization of 1 detected at $2 \mathrm{~K}$. The red solid line shows the Brillouin curve corresponding to two decoupled Fe(II) ions. The blue solid line is the output of the molecular field model calculation for an antiferromagnetically coupled chain of the Fe(II) ions.

\subsubsection{Modeling}

The pertinent model should duly reflect the structural features of the studied compound $\mathbf{1}$. There is one constitutive part of its crystal structure, i.e. the rectangular sheets of $\mathrm{Fe}$ (II) ions involving two types of linkages: the double -N-C-N-C-N- linkage resulting in the averaged Fe-Fe distance of $7.472 \AA$ and the 4,4'-bipyridine linkage resulting in the averaged Fe-Fe distance of $11.557 \AA$. The first linkage joins the $\mathrm{Fe}(\mathrm{II})$ ions along the $b$ crystallographic axis, while the Fe chains formed by the second linkage are located in the $a c$ plane. Because the distance between the Fe(II) ions in the $b$ crystallographic direction are substantially smaller, we assume that the superexchange coupling between the $\mathrm{Fe}$ (II) ions mediated through the bipyridine bridges is relatively smaller and can be neglected, so that the magnetic properties of the studied compound can be explained by considering that the compound consists of Fe chains exchange coupled through the double $-\mathrm{N}-\mathrm{C}-\mathrm{N}-\mathrm{C}-\mathrm{N}-$ bridges.

As the spins of the $\mathrm{Fe}(\mathrm{II})$ ions are relatively high, $S_{\mathrm{Fe}}=2$, one can venture a classical approach to the magnetic properties of the constitutive chains. Within this approach the spin of the Fe(II) ion may be treated as a classical vector. An analytical expression for the magnetic susceptibility of an infinite chain of classical spins has been derived by Fisher [Fisher]. The corresponding expression, in which the classical spin has been scaled to a real quantum spin, reads

$$
\chi_{\mathrm{F}}(T ; J, g, S)=\frac{N_{\mathrm{A}} \mu_{\mathrm{B}}^{2} g^{2} S(S+1)}{3 k_{\mathrm{B}} T} \frac{1+u(J, S, T)}{1-u(J, S, T)}
$$

where

$$
u(J, S, T)=\operatorname{coth}\left[\frac{J S(S+1)}{k_{\mathrm{B}} T}\right]-\left[\frac{k_{\mathrm{B}} T}{J S(S+1)}\right]
$$

No analytical calculation is possible for the field dependence of the isothermal magnetization for such a chain. At the same time, an exact quantification of the chain model would require nontrivial and time-consuming Monte Carlo simulations, we therefore resort here to a simplified approach based on the molecular field approximation (MFA). As the $\chi T$ signal in Fig. 1 implies an antiferromagnetic coupling between the Fe(II) ions, we shall assume that the correlated chain consists of two sublattices A1 and A2 with stoichiometric factors $v_{1}$ and $v_{2}$ respectively. Sublattices $\mathrm{A} 1$ and A2 are occupied by the A type ions with spin $S_{\mathrm{A}}$ and the Landé factor $g_{\mathrm{A}}$. Thus the system may be described by the formula $\mathrm{A}_{v_{1}} \mathrm{~A} 2_{v_{2}}$. The Hamiltonian pertinent to the system is given by the following formula 


$$
\hat{H}=-J \sum_{(i j)} \hat{S}_{\mathrm{A} 1 i} \cdot \hat{S}_{\mathrm{A} 2 j}+\mu_{\mathrm{B}} g_{\mathrm{A}} \sum_{i}\left(\hat{S}_{\mathrm{A} 1 i}+\hat{S}_{\mathrm{A} 2 i}\right) \cdot \vec{H}
$$

where $(i j)$ denotes the summation over the pairs of the nearest neighbors, $J$ is the superexchange coupling constant, $\mu_{\mathrm{B}}$ is the Bohr magneton, and $\vec{H}$ is the external magnetic field. In the framework of MFA the Hamiltonian in Eq. (3) is reduced to the following form

$$
\hat{H}=g_{\mathrm{A}} \mu_{\mathrm{B}} \hat{S}_{\mathrm{A} 1} \cdot \vec{H}_{\mathrm{A} 1}+g_{\mathrm{B}} \mu_{\mathrm{B}} \hat{S}_{\mathrm{A} 2} \cdot \vec{H}_{\mathrm{A} 2},
$$

where the molecular fields $\vec{H}_{\mathrm{X}}(\mathrm{X}=\mathrm{A} 1, \mathrm{~A} 2)$ read

$$
\begin{aligned}
& \vec{H}_{\mathrm{A} 1}=\vec{H}+\Lambda_{\mathrm{A} 1 \mathrm{~A} 2} \vec{M}_{\mathrm{A} 2} \\
& \vec{H}_{\mathrm{A} 2}=\vec{H}+\Lambda_{\mathrm{A} 2 \mathrm{~A} 1} \vec{M}_{\mathrm{A} 1}
\end{aligned}
$$

Quantity $\vec{M}_{\mathrm{X}}$ denotes the molar magnetization of sublattice X, and the molecular field constants $\Lambda_{\mathrm{XY}} \mathrm{read}$

$$
\Lambda_{\mathrm{A} 1 \mathrm{~A} 2}=\frac{J Z_{\mathrm{A} 1 \mathrm{~A} 2}}{N_{\mathrm{A}} \mu_{\mathrm{B}}^{2} \nu_{\mathrm{A} 2} g_{\mathrm{A}}^{2}}, \quad \Lambda_{\mathrm{A} 2 \mathrm{~A} 1}=\frac{J Z_{\mathrm{A} 2 \mathrm{~A} 1}}{N_{\mathrm{A}} \mu_{\mathrm{B}}^{2} \nu_{\mathrm{A} 1} g_{\mathrm{A}}^{2}},
$$

where $Z_{\mathrm{XY}}$ denotes the number of the nearest neighbor $\mathrm{Y}$ type ions of the $\mathrm{X}$ type ion. It is clear that the number of the coupling connections between the A1 and A2 type ions in a mole of the compound may be written either as $N_{\mathrm{A}} v_{1} Z_{\mathrm{A} 1 \mathrm{~A} 2}$ or as $N_{\mathrm{A}} v_{2} Z_{\mathrm{A} 2 \mathrm{~A} 1}$, hence $v_{1} Z_{\mathrm{A} 1 \mathrm{~A} 2}=v_{2} Z_{\mathrm{A} 2 \mathrm{~A} 1}$. The above relations and Eq. (6) imply that $\Lambda_{\mathrm{A} 1 \mathrm{~A} 2}=\Lambda_{\mathrm{A} 2 \mathrm{~A} 1} \equiv \Lambda$, thus there a single independent molecular field constants in the model $\Lambda$. For an arbitrary thermodynamic point $(T, H)$ the molecular field model defined in Eqs. (2)-(4) should be solved by an iterative numerical method. However, in the special case where the temperature is high compared to $J / k_{\mathrm{B}}\left(k_{\mathrm{B}}\right.$-the Boltzmann constant) one can venture to calculate the magnetic susceptibility $\chi$ of the system. An additional simplification stems from the fact that the model is isotropic (all exchange interactions are of the isotropic Heisenberg type) and the scalar counterpart of Eq. (3) can be used. Then the system is in the paramagnetic state and the molar magnetizations are directly proportional to the magnetic field, i.e. $M_{\mathrm{x}}=\chi_{\mathrm{x}} H_{\mathrm{x}}$, where

$$
\chi_{\mathrm{X}}=\frac{N_{\mathrm{A}} \mu_{\mathrm{B}}^{2} \nu_{\mathrm{X}} g_{\mathrm{X}}^{2} S_{\mathrm{X} 0}\left(S_{\mathrm{X} 0}+1\right)}{3 k_{\mathrm{B}} T}
$$

is the paramagnetic molar susceptibility of sublattice $\mathrm{X}$. The system of linear equations $M_{\mathrm{X}}=\chi_{\mathrm{X}} H_{\mathrm{X}}$ (X=A1, A2) can be solved by the Cramer method for $M_{\mathrm{X}}$ and the total susceptibility of the system can be calculated as $\chi=\left(M_{\mathrm{A} 1}+M_{\mathrm{A} 2}\right) / H$ :

$$
\chi=\frac{\chi_{\mathrm{A} 1}+\chi_{\mathrm{A} 2}+2 \chi_{\mathrm{A}} \chi_{\mathrm{B}} \Lambda}{1-\chi_{\mathrm{A} 1} \chi_{\mathrm{A} 2} \Lambda^{2}}
$$

To compute the total molar magnetization $M_{\text {total }}$ one should iteratively solve a set of coupled equations for the thermally averaged values of sublattice spins 


$$
\begin{aligned}
& \left\langle S_{\mathrm{A} 1}\right\rangle=S_{\mathrm{A} 0} B_{S_{\mathrm{A} 0}}\left[\frac{g_{\mathrm{A}} \mu_{\mathrm{B}} S_{\mathrm{A} 0} H}{k_{\mathrm{B}} T}+\frac{J Z_{\mathrm{A} 1 \mathrm{~A} 2} S_{\mathrm{A} 0}}{k_{\mathrm{B}} T}\left\langle S_{\mathrm{A} 2}\right\rangle\right], \\
& \left\langle S_{\mathrm{A} 2}\right\rangle=S_{\mathrm{A} 0} B_{S_{\mathrm{A} 0}}\left[\frac{g_{\mathrm{A}} \mu_{\mathrm{B}} S_{\mathrm{A} 0} H}{k_{\mathrm{B}} T}+\frac{J Z_{\mathrm{A} 2 \mathrm{~A} 1} S_{\mathrm{A} 0}}{k_{\mathrm{B}} T}\left\langle S_{\mathrm{A} 1}\right\rangle\right],
\end{aligned}
$$

where $B_{S}$ is the Brillouin function, $S_{\mathrm{A} 0}$ is the value of $\left\langle S_{\mathrm{A} 1}\right\rangle$ and $\left\langle S_{\mathrm{A} 2}\right\rangle$ at $\mathrm{T}=0 \mathrm{~K}$. The total magnetization is then given by the formula

$$
M_{\text {total }}=N_{\mathrm{A}} \mu_{\mathrm{B}}\left[v_{1} g_{\mathrm{A}}\left\langle S_{\mathrm{A} 1}\right\rangle+v_{2} g_{\mathrm{A}}\left\langle S_{\mathrm{A} 2}\right\rangle\right] .
$$

In the case under study sublattices $\mathrm{A} 1$ and $\mathrm{A} 2$ consist both of the $\mathrm{Fe}(\mathrm{II})$ ions. The pertinent values of parameters are: $v_{1}=1, v_{2}=1, Z_{\mathrm{Fe} 1 \mathrm{Fe} 2}=2, Z_{\mathrm{Fe} 2 \mathrm{Fel}}=2, S_{\mathrm{Fe} 0}=2$.

The experimental $\chi T$ value at $300 \mathrm{~K}$ amounts to $6.242 \mathrm{~cm}^{3} \mathrm{~K} \mathrm{~mol}^{-1}$. On cooling the $\chi T$ signal slightly increases attaining the maximum of $6.585 \mathrm{~cm}^{3} \mathrm{~K} \mathrm{~mol}{ }^{-1}$ at $91.6 \mathrm{~K}$, and then decreases reaching the value of $2.355 \mathrm{~cm}^{3} \mathrm{~K} \mathrm{~mol}^{-1}$ at $2 \mathrm{~K}$. The decreasing trend of the $\chi T$ signal on heating the sample may be attributed to some diamagnetic correction originating from the sample itself and from the mounting setup (plastic straw). The isothermal magnetization (see Fig. 2) does not show hysteretic behavior and is a smoothly increasing function of the applied field reaching the value of $5.53 N_{\mathrm{A}} \mu_{\mathrm{B}}$ in the field of $7 \mathrm{~T}$. To get a more quantitative insight into the details of the intrachain interactions we fitted function

$$
\chi=2 \chi_{\mathrm{F}}\left(T ; J, g_{\mathrm{Fe}}, 2\right)+\chi_{0},
$$

where the factor 2 is introduced to account for the stoichiometry of the formula unit, to the experimental susceptibility data. The best fit yields $J=-0.450(3) \mathrm{cm}^{-1}, g_{\mathrm{Fe}}=2.150(1)$, and $\chi_{0}=-$ $0.00198(4) \mathrm{cm}^{3} \mathrm{~mol}^{-1}$. Thus, the intrachain coupling between the Fe(II) ions is relatively weak and antiferromagnetic. The fit is depicted by the red solid line in Fig. 1. The corresponding agreement quotient for susceptibility based on the mean square deviation from the experimental points is $Q_{\chi T}=\sum\left[(\chi T)_{\text {obs }}-(\chi T)_{\text {calc }}\right]^{2} / \sum\left[(\chi T)_{\text {obs }}\right]^{2}=3.0 \cdot 10^{-5}$. Next, we fitted the Curie-Weiss law, appended with the diamagnetic correction $\chi_{0}$ found in the first fit, to the temperature dependence of the inverse susceptibility:

$$
\chi=\frac{C}{T-\theta}+\chi_{0}
$$

The best fit yielded $C=6.916(3) \mathrm{cm}^{3} \mathrm{~K} \mathrm{~mol}^{-1}$ and $\theta=-2.27(9) \mathrm{K}$. It is depicted with the red solid line in Inset of Fig. 1. One can see that the agreement with the experimental data is satisfactory. The value of the Curie constant implies $g_{\mathrm{Fe}}=2.147$, which is in perfect agreement with value found in the first fit. Eq. (8) establishes the following relation between the Weiss temperature $\theta$ and the intrachain coupling constant $J, J=1 / 4 k_{\mathrm{B}} \theta$, which yields $J=-0.39 \mathrm{~cm}^{-1}$ being roughly consistent with the value implied by the first fit. The necessary difference may be ascribed to the fact that the first fit involved the Fisher expression accounting rigorously for the infinitely long spin chain, while the Curie-Weiss expression used in the second fit corresponds to the approximate molecular field model.

For the sake of comparison with experiment the isothermal magnetization was calculated by two independent methods. Firstly, it was assumed to be the sum of the Brillouin functions (purely paramagnetic contribution) corresponding to two non-interacting $\mathrm{Fe}(\mathrm{II})$ ions with the Landé factor 
implied by the first susceptibility fit. It is depicted by the red solid line in Fig. 2. It can be seen that the experimental values lie deep below this curve. The second method consisted in solving iteratively the set of equations in Eq. (9), with the parameters implied by the first susceptibility fit, and calculating the magnetization according to the formula in Eq. (10). This method was meant to account for the antiferromagnetic intrachain coupling between the Fe(II) ions. The result is depicted by the blue solid line in Fig. 2. It is apparent that the magnetization values are reproduced only in the low-field regime. For higher fields the MFA-based curve exceeds the experimental values and it finally saturates. This indicates that the molecular field approximation is too crude to duly reproduce the short-range correlations of the exchange-coupled spin chain suppressing considerably the magnetization values.

\section{Conclusion}

In this work, synthesis, single crystal X-ray structure analyses and characterization of a new dicyanamide bridged Fe(II) coordination polymers, 1, containing 4,4'-bipyridine ligand, have been reported. The structural analysis of the complexe shows that the environment around the sixcoordinated Fe(II) ion can be described as a slightly distorted octahedral geometry and the dca ligand is coordinated to the metal site in a bidentate $\mu_{1,5}$-bridging mode to form 1-D coordination polymer. These polymeric chains are also linked with bpy ligands to form 2-D layers. The selection of 4,4'-bpy as co-ligand has a rather important role in the crystal structure arrangement of dca coordination polymers due to its bridging ability.

The studied compound may be treated as a weakly interacting assembly of linear chains of the $\mathrm{Fe}(\mathrm{II})$ ions with the antiferromagnetic intrachain coupling.

\section{References}

[1] C. Genre, E. Jeanneau, A. Bousseksou, D. Luneau, S. A. Borshth, and G. S. Matouzenko, Chem. - A Eur. J., 14, 697-705, (2008).

[2] J. Carranza, C. Brennan, J. Sletten, F. Lloret, and M. Julve, J. Chem. Soc. Dalt. Trans., 3164-3170, (2002).

[3] Q. Li and H.-T. Wang, Acta Crystallogr. Sect. C Struct. Chem., 70, 1054-1056, (2014).

[4] K. R. Dunbarb, R. March, and A. April, 954-958, (2001).

[5] S. Benmansour, C. Atmani, F. Setifi, S. Triki, M. Marchivie, C.J. G_omez-García, Coord. Chem. Rev. 254 (2010) 1468-1478.

[6] S. Benmansour, F. Setifi, S. Triki, J.-Y. Salaün, F. Vandevelde, J. Sala-Pala, C.J. G_omez-García, T. Roisnel, Eur. J. Inorg. Chem. 2007 (2007) 186-194.

[7] A. Miyazaki, K. Okabe, T. Enoki, F. Setifi, S. Golhen, L. Ouahab, T. Toita, J. Yamada, Synth. Met. 137 (2003) 1195-1196.

[8] F. Setifi, E. Milin, C. Charles, F. Th_etiot, S. Triki, C.J. G_omez-García, Inorg. Chem. 53 (2014) 97-104.

[9] Z. Setifi, F. Setifi, L. El Ammari, M. El-Ghozzi, J. Sopkov_a-de Oliveira Santos, H. Merazig, C. Glidewell, Acta Crystallogr. Section C Struct. Čhem. 70 (2014) 19-22. [10] C. Yuste, A. Bentama, N. Marino, D. Armentano, F. Setifi, S. Triki, F. Lloret, M. Julve, Polyhedron 28 (2009) 1287-1294.

[11] S. Benmansour, F. Setifi, S. Triki, C.J. G_omez-García, Inorg. Chem. 51 (2012) 2359-2365.

[12] E. Gungor, Y. Yahsi, H. Kara, A. Caneschi, CrystEngComm 17 (2015) 3082-3088.

[13] U. Erkarslan, G. Oylumluoglu, M.B. Coban, E. €Oztürk, H. Kara, Inorg. Chim. Acta. 445 (2016) 57-61.

[14] H. Kara, A. Azizoglu, A. Karaoglu, Y. Yahsi, E. Gungor, A. Caneschi, L. Sorace, Cryst EngComm 14 (2012) 7320.

[15] Z. Setifi, B. Gaamoune, H. Stoeckli-Evans, D.-A. Rouag, F. Setifi, Acta Crystallogr. Sect. C Cryst. Struct. Commun. 66 (2010) m286-m289.

[16] Z. Setifi, M. Ghazzali, C. Glidewell, O. P_erez, F. Setifi, C.J. G_omez-García, 


\section{A. ADDALE et al./ISITES2018 Alanya - Antalya - Turkey}

J. Reedijk, Polyhedron 117 (2016) 244-248.

[17] A. M. Kutasi, A. R. Harris, S. R. Batten, B. Moubaraki, and K. S. Murray, Cryst. Growth Des., 4, 605-610, (2004).

[18] J. L. Manson, A. M. Arif, C. D. Incarvito, L. M. Liable-Sands, A. L. Rheingold, and J. S. Miller, J. Solid State Chem., 145, 369-378, (1999).

[19] A. Claramunt, A. Escuer, F. a. Mautner, N. Sanz, and R. Vicente, J. Chem. Soc. Dalt. Trans., 2627-2630, (2000).

[20] J. L. Manson, D. W. Lee, A. L. Rheingold, and J. S. Miller, Inorg. Chem., 37, 59665967, (1998).

[21] J. L. Manson, C. D. Incarvito, A. L. Rheingold, and J. S. Miller, J. Chem. Soc. Dalt. Trans., 3705-3706, (1998).

[22] P. Jensen et al., J. Solid State Chem., 145, 387-393, (1999).

[23] J. L. Manson, A. M. Arif, and J. S. Miller, J. Mater. Chem., 9, 979-983, (1999).

[24] S. R. Batten et al., J. Chem. Soc. Dalt. Trans., 2987-2997, (1999).

[25] S. R. Batten and K. S. Murray, Coord. Chem. Rev., 246, 103-130, (2003).

[26] A. Escuer, F. A. Mautner, N. Sanz, and R. Vicente, Inorg. Chem., 39, 1668-1673,(2000).

[27] S. Triki, F. Thétiot, J.-R. Galán-Mascarós, J. Sala Pala, and K. R. Dunbar, New J. Chem.,

25, 954-958, (2001).

[28] S. Konar, U. Saha, M. Dolai, and S. Chatterjee, J. Mol. Struct., 1075, 286-291, (2014).

[29] M. Biswas, S. R. Batten, P. Jensen, and S. Mitra, Aust. J. Chem., 59, 115-117, (2006).

[30] Bruker APEX2, SADABS and SAINT, Bruker AXS Inc., Madison, Wisconsin, USA,

(2013).

[31] G. M. Sheldrick, Acta Cryst. A64 (2008) 112-122.

[32] G. M. Sheldrick, Acta Cryst. C71 (2015) 3-8.

[33] J.-H. Luo, M.-C. Hong, R. Cao, Y.-C. Liang, Y.-J. Zhao, R.-H. Wang, J.-B. Weng, Polyhedron 21 (2002) 893-898.

[34] F. Reuter, E. Rentschler, Polyhedron 52 (2013) 788-796.

[35] F. Setifi, P. Konieczny, C. Glidewell, M. Arefian, R. Pelka, Z. Setifi, M. Mirzaei,

J. Mol. Struct. 1149 (2017) 149-154.

[36] Z. Setifi, F. Setifi, S.W. Ng, A. Oudahmane, M. El-Ghozzi, D. Avignant, Acta Crystallogr. Section E Struct. Rep. 69 (2013) m12-m13.

[37] Z. Setifi, K.V. Domasevitch, F. Setifi, P. Mach, S.W. Ng, V. Pet_rí_cek, M. Du_sek, Acta Crystallogr. Sect. C Cryst. Struct. Commun. 69 (2013) 1351-1356.

[38] Z. Setifi, F. Setifi, H. Boughzala, A. Beghidja, C. Glidewell, Acta Crystallogr. Section C Struct. Chem. 70 (2014) 465-469.

[39] R.D. Shannon, C.T. Prewitt, Acta Crystallogr. Sect. B Strct. Crystallogr. Cryst. Chem. 25 (1969) 925-946. 